\title{
Aberrant overexpression of vascular endothelial growth factor in pancreatic ductal adenocarcinoma is associated with aggressive clinical behavior
}

\author{
This article was published in the following Dove Press journal: \\ Pathology and Laboratory Medicine International \\ 12 July 2010 \\ Number of times this article has been viewed
}

\author{
Naomi Y Jiang' \\ Bruce A Woda ${ }^{2}$ \\ Liping Zhang ${ }^{2}$ \\ Suyang $\mathrm{HaO}^{2}$ \\ Karen A Dresser ${ }^{2}$ \\ Di Lu ${ }^{2}$ \\ 'Massachusetts Institute of \\ Technology, Worcester, MA, USA; \\ ${ }^{2}$ Department of Pathology, University \\ of Massachusetts Medical Center, \\ Worcester, MA, USA
}

\begin{abstract}
Pancreatic adenocarcinoma is a leading cause of cancer-related deaths in the United States. In this study, we studied vascular endothelial growth factor (VEGF) expression in pancreatic adenocarcinoma by immunohistochemical staining. Clinical follow-up and survival data were analyzed. We determined that VEGF was aberrantly overexpressed in a subset of primary pancreatic adenocarcinoma. Statistically, VEGF overexpression was associated with higher stage, higher grade, and lymph node metastasis $(P<0.001, P=0.012$, and $P<0.005$, respectively). Additionally, patients of this subset had a much shorter overall survival than patients without VEGF overexpression, as evidenced by Kaplan-Meier plots and the log-rank test $(P=0.001)$. The 5 -year overall survival rate was $17 \%$ in patients with VEGF overexpression compared to $52 \%$ in patients without VEGF overexpression. The median survival was only 13 months for patients with VEGF overexpression compared to 65 months for patients without. In conclusion, VEGF is a biomarker that identifies a subset of pancreatic ductal adenocarcinoma with aggressive clinical behavior.
\end{abstract}

Keywords: pancreatic adenocarcinoma, VEGF, cancer

\section{Introduction}

Pancreatic ductal adenocarcinoma is the fourth leading causes of cancer-related deaths in the United States. ${ }^{1,2}$ Outcome data shows that once recognized, patients have a mean survival time of 6 months and a 5-year survival rate of only $4 \%{ }^{3}$ The only form of curative treatment is surgical resection of early stage pancreatic adenocarcinoma. ${ }^{4}$ However, $80 \%$ of these tumors recurred within two years of surgery. ${ }^{5}$

More recent studies have shown improved survival. Various adjuvant and neoadjuvant treatments have been developed and are used in conjunction with surgical resection. ${ }^{6}$ Retroperitoneal resection has been advocated to attempt to eliminate lymph node metastasis. ${ }^{7}$ Systemic chemotherapy has emerged as an effective option to treat this cancer. ${ }^{8}$ Antivascular endothelial growth factor (anti-VEGF) antibody to treat advanced pancreatic adenocarcinoma as a mean of inhibition of neoangiogenesis has also been developed and is in clinical trials. ${ }^{9}$ These approaches have shown promising efficacy that may substantially prolong patient's survival. To improve outcome further, biomarkers have attracted much attention with the hope that these markers can predict tumor behavior and also serve as therapeutic targets, thus providing tools for the individualized treatment of pancreatic adenocarcinoma. ${ }^{10}$
Correspondence: Di Lu Department of Pathology Three Biotech, One Innovation Drive, Worcester, MA 01605, USA $\mathrm{Tel}+\mathrm{I} 5087936147$

Fax +I 5087936110

Email lud@ummhc.org 
Vascular endothelial growth factor (VEGF), also known as VEGF-A, is a member of a group of related genes consisting of four isoforms, which carry out diverse cellular and biological activities. ${ }^{11}$ It is thought that VEGF is important in promoting malignant progression. ${ }^{12,13}$ We now know that VEGF asserts such actions via at least three mechanisms. First, VEGF stimulates neoangiogenesis by a paracrine mechanism in which the tumor cells express and secrete high level of VEGF that binds to VEGF receptors present on the surface of endothelial cells. ${ }^{14}$ VEGF can also stimulate tumor growth as an autocrine growth factor by binding to the receptors on its own cell surface. ${ }^{15}$

When VEGF binds to intracellular receptors within tumor cells, it promotes tumor cell survival, an intracrine mechanism. ${ }^{16}$ The level of VEGF expression by tumor cells therefore is speculated to influence the tumor behavior. In the present study, we examined the status of VEGF expression in tumor tissues of surgically resected human pancreatic adenocarcinoma, and analyzed the relationship between the expression of VEGF with tumor differentiation, tumor stage, tumor metastasis, and overall survival.

\section{Materials and methods \\ Patients and tumor samples}

Forty-five patients underwent Whipple resections for pancreatic adenocarcinoma, ductal type, between 2000-2007 at the UMass Memorial Medical Center, Worcester, MA. The tumor slides and corresponding tissue blocks were obtained from our archives. Routine formalin-fixed, paraffinembedded sections were reviewed and two pathologists confirmed the diagnoses. Staging was done according to the American Joint Committee on Cancer's Cancer Staging Manual, 6th edition. ${ }^{17}$ By use of the tumor (T) node (N) metastasis (M) classification system, we identified two stage IA tumors, four stage IB tumors, 11 stage IIA tumors, 19 stage IIB tumors, five stage III tumors, and three stage IV tumors. Lymph node metastasis was found in samples from $25(56 \%)$ of 45 patients. Clinical follow-up was obtained by reviewing the patient's medical record. Overall survival was measured from the date of tumor resection to the date of death or was censored as of the date of the last follow-up visit for survivors. The Institutional Review Board at our institution approved this study.

\section{Immunohistochemical analysis}

Immunohistochemical studies were performed on 5- $\mu \mathrm{m}$ sections of formalin-fixed, paraffin-embedded tissue as previously described. ${ }^{18}$ The analysis was done blinded by two pathologists. The tissue blocks that were most representative and showed highest tumor volume were selected for staining. Antigen retrieval was carried out with $0.01 \mathrm{~mol} / \mathrm{L}$ citrate buffer at $\mathrm{pH} 6.0$, in an 800-W microwave oven for 15 minutes before immunostaining. The slides were stained on the DAKO Autostainer (DAKO Corporation, Carpinteria, CA) using the EnVision (Dako) staining reagents.

The sections were blocked for endogenous protein binding and peroxidase activity with an application of Dual Endogenous Block (Dako) for 10 minutes, followed by a buffer wash. The sections were then incubated with a monoclonal antibody specific for VEGF (clone SP28; Labvision, Fremont, CA) at a 1:25 dilution for 30 minutes, followed by a buffer wash. Sections were then incubated with the EnVision + Dual Link reagent (a polymer conjugated with goat-anti-mouse-Ig, and horseradish peroxidase) for 30 minutes. The sections were then washed, and treated with diaminobenzidine (DAB) and hydrogen peroxide, to produce the visible end product. A toning solution (DAB Enhancer; Dako) was used to enrich the final color. The sections were counterstained with hematoxylin, dehydrated and coverslipped with mounting media. Negative control sections were stained by replacing the primary antibody with nonimmune mouse immunoglobulin G (IgG; Vector, Burlingame, CA) at 2.0 a concentration of $2 \mu \mathrm{g} / \mathrm{mL}$. Positivity of the primary antibody was defined as strong brown staining in the cytoplasm, which is most concentrated in the perinuclear region. Negative staining was defined as no staining, or weak diffuse background granules. For carcinoma with VEGF overexpression, each tumor had at least $20 \%$ of the tumor cells that exhibited positive staining. The intensity of the staining has mild variation within the same tumor, but the average staining intensity among all VEGF positive tumors was similar. In contrast, for carcinoma without VEGF overexpression, essentially no tumor cell was stained positive.

\section{Statistical analysis}

Age, sex, size of the tumor, tumor stage, and tumor differentiation were collected as baseline variables. The distribution of each baseline variable was compared for VEGF-positive and VEGF-negative subgroups with the Wilcoxon rank sum test for continuous variables and the Fisher's exact test for categorical variables. Overall survival was estimated by the Kaplan - Meier method and evaluated with the use of logrank test for univariate analysis.

\section{Results}

Sixteen (36\%) of the 45 cases of pancreatic adenocarcinoma showed VEGF overexpression (Figure 1) with at least 20\% 


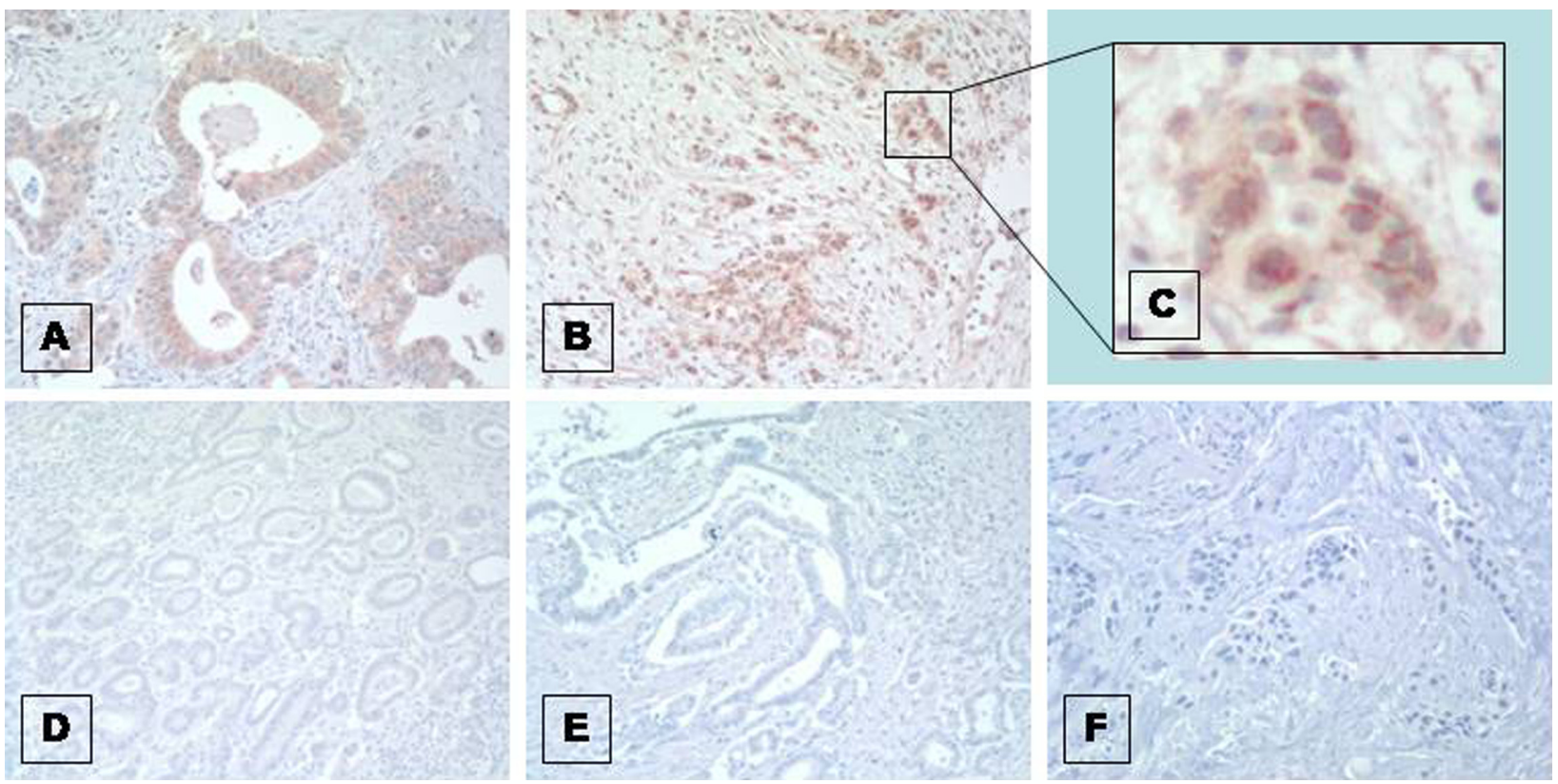

Figure I Representative positive (A, B, C) and negative (D, E, F) immunohistochemical stains of VEGF in the peri-nuclei region and cytoplasm of well (D), moderately (A, E), and poorly (B, C, F) differentiated pancreatic ductal adenocarcinomas (Magnification 200X).

Abbreviation: VEGF, vascular endothelial growth factor.

positive cells for each case. The remaining 29 cases were negative. Table 1 provides the VEGF status and the relevant clinical characteristics of the 45 patients with pancreatic adenocarcinoma. Age, gender, and tumor size were not associated with VEGF-positivity $(P=0.923, P=0.068$, and $P=0.078$, respectively). Positive VEGF staining was associated with higher tumor stage, higher grade and lymph node metastasis $(P<0.001, P=0.012$, and $P<0.005$, respectively), which are established pathological predictors of clinical outcome for pancreatic adenocarcinoma.

None of the stage IA, IB, and IIA tumors was VEGFpositive while $58 \%$ of stage IIB, $60 \%$ of stage III, and $67 \%$ of stage IV were VEGF-positive (Figure 2). All tumors which showed VEGF expression had lymph node metastasis, whereas none of the tumors without lymph node metastasis was VEGF-positive (Figure 3A). Among all patients with metastasis, $64 \%$ of the primary tumors were VEGF-positive (Figure 3B). Therefore, as a marker to detect tumor lymph node metastasis, the positive staining for VEGF protein in primary pancreatic adenocarcinoma has a sensitivity of $64 \%$, a specificity of $100 \%$, a positive predictive value of $100 \%$, and a negative predictive value of $69 \%$.

Kaplan - Meier plots and log-rank tests of survival (Figure 4) in patients with pancreatic adenocarcinoma showed that patients with negative VEGF staining in their primary tumor had significant longer overall survival than patients with positive VEGF staining (Figure 4; $P=0.001$ ). The 5-year overall survival rate was $52 \%$ in patients with negative VEGF staining versus $17 \%$ in patients with positive VEGF staining. The median survival was 65 months in patients with negative VEGF staining, whereas the median survival was only 13 months in patients with positive VEGF staining in their primary tumors.

\section{Discussion}

Normal pancreatic ductal epithelial cells express low levels of VEGF, which are not detected by the immunohistochemical staining method used in this study. The positive cytoplasmic staining of pancreatic adenocarcinoma cells illustrated in this study represent aberrant overexpression of VEGF protein. In the present study, we demonstrated that the overexpression of VEGF is a biomarker which identifies a subset of pancreatic ductal adenocarcinoma characterized by aggressive clinical behavior. We provide evidence that overexpression of VEGF is associated with the presence of lymph node metastasis and advanced cancer stages specifically stages IIB-IV, and that patient with VEGF positive cancer had a much-shortened overall survival.

VEGF overexpression has been demonstrated in various cancers including pancreatic carcinomas. ${ }^{19-21}$ Prior study concluded that nearly all pancreatic ductal adenocarcinomas overexpressed VEGF at variable degrees judged as 1 to 3 plus staining intensity. ${ }^{22}$ Our study indicates that only 
Table I Clinical and pathological data of pancreatic adenocarcinoma shown by VEGF immunohistochemical staining results

\begin{tabular}{|c|c|c|c|}
\hline & $\begin{array}{l}\text { VEGF-positive } \\
\text { tumors }(n=16)\end{array}$ & $\begin{array}{l}\text { VEGF-negative } \\
\text { tumors }(n=29)\end{array}$ & $P$ value \\
\hline \multicolumn{4}{|l|}{ Sex } \\
\hline Female & $6(29 \%)$ & I5 (7I\%) & 0.068 \\
\hline Male & $10(42 \%)$ & $14(58 \%)$ & \\
\hline Age (years) & & & 0.923 \\
\hline Mean (SD) & $64.8(10.4)$ & $66.2(11.7)$ & \\
\hline Tumor differentiation & & & 0.012 \\
\hline Well & $0(0 \%)$ & $10(100 \%)$ & \\
\hline Moderate & $8(38 \%)$ & $13(62 \%)$ & \\
\hline Poor & $9(64 \%)$ & $5(36 \%)$ & \\
\hline Tumor stage & & & $<0.001$ \\
\hline IA & 0 & $2(100 \%)$ & \\
\hline IB & 0 & $4(100 \%)$ & \\
\hline IIA & 0 & II (I00\%) & \\
\hline IIB & II (58\%) & $8(42 \%)$ & \\
\hline III & $3(60 \%)$ & $2(40 \%)$ & \\
\hline IV & $2(67 \%)$ & I (33\%) & \\
\hline Tumor size (cm) & & & 0.078 \\
\hline Mean (SD) & $4.8(3.6)$ & $3.2(2.8)$ & \\
\hline Tumor metastasis & & & 0.005 \\
\hline No & 0 & $20(100 \%)$ & \\
\hline Yes & $16(64 \%)$ & $9(36 \%)$ & \\
\hline Lymph node numbers & & & 0.914 \\
\hline Median & 16 & 15 & \\
\hline Mean (SD) & $16.23(11.02)$ & $15.60(12.65)$ & \\
\hline
\end{tabular}

Abbreviations: SD, standard deviation; VEGF, vascular endothelial growth factor.

a portion of these carcinomas actually exhibits aberrant VEGF overexpression. The remaining tumors do express VEGF, but at the same expression levels of normal pancreatic ductal cells. We worked up our staining protocol to such that these normal expressions are not detected. The mechanisms that regulate aberrant VEGF overexpression are not well understood. ${ }^{11}$ The presence of VEGF overexpression varied among different types of tumors. VEGF overexpression in malignant neoplasms has been linked to worse prognoses. ${ }^{22-24}$ This study further demonstrates that VEGF overexpression is associated with aggressive clinical behavior of pancreatic ductal adenocarcinomas.

The application of VEGF as a prognostic marker may improve clinical management. The overexpression of VEGF has $100 \%$ specificity for the presence of lymph node metastasis. This is very useful, since lymph node removal

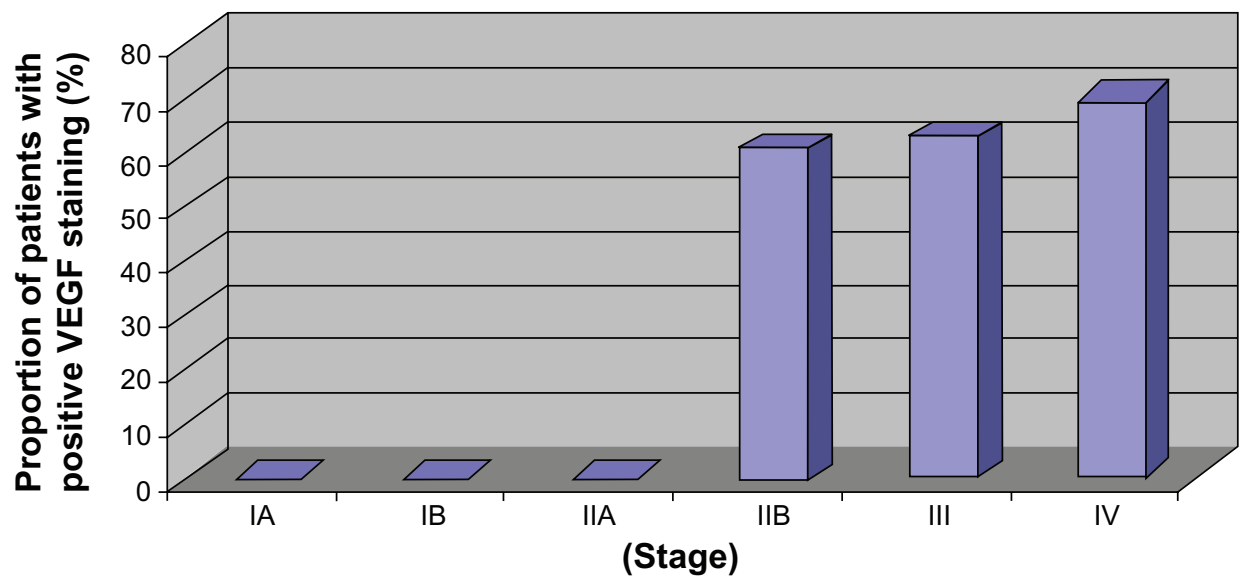

Figure 2 Association of VEGF positive staining with tumor stage. Abbreviation: VEGF, vascular endothelial growth factor. 

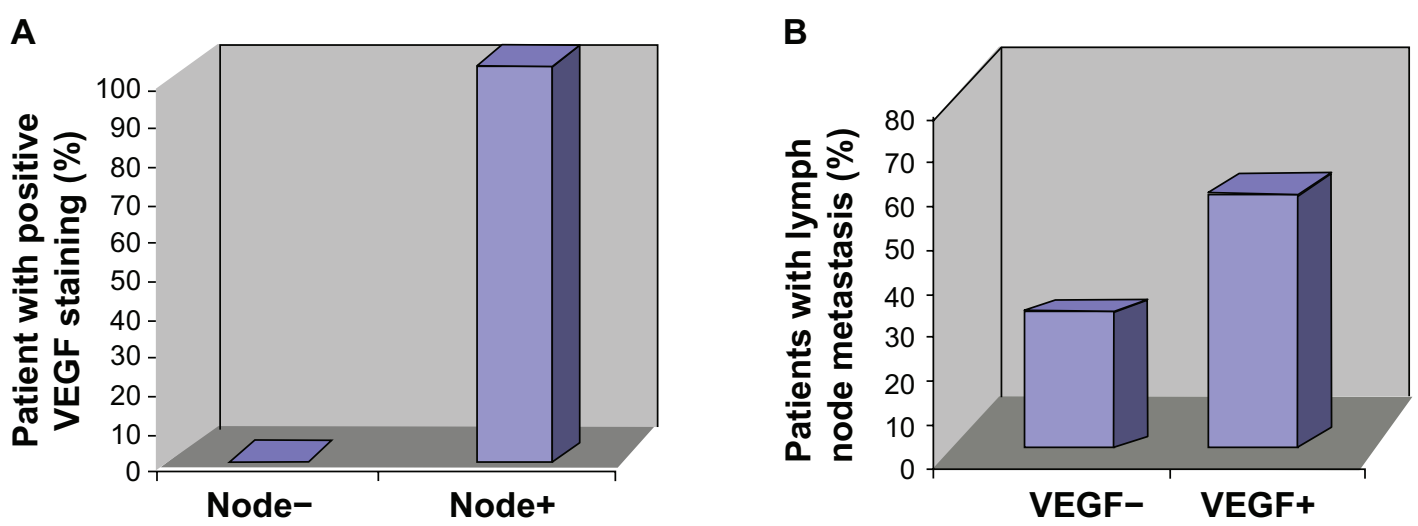

Figure 3 A) All patients with tumors stained positive for VEGF had lymph node metastasis. B) $64 \%$ of patients with lymph node metastasis had tumors stained positive for VEGF. Abbreviations: VEGF, vascular endothelial growth factor.

during surgical resection of pancreatic adenocarcinoma may be challenging, and inadequate lymph node dissection may lead to false negative lymph node status and under-staging of the tumor.

Furthermore, surgical retroperitoneal lymph node dissection in addition to conventional resection of the tumor, has been advocated in recent years, despite the fact that such procedure may lead to severe complications. The ability of VEGF to identify patients with lymph node metastasis can assist in individualized decision making and treatment options, and assist in selectively applying retroperitoneal dissection to those who may benefit.

Recently, the anti-VEGF agent, bevacizumab, a humanized recombinant monoclonal anti-VEGF antibody, has been used in clinical trials to treat patients with pancreatic adenocarcinomas, and so far have not achieved the effectiveness anticipated. ${ }^{25,26}$ In this study, only $36 \%$ of ductal carcinoma of pancreas has VEGF overexpression. It may be speculated that anti-VEGF agent may be more effective or effective only, for tumors with VEGF overexpression. Further studies are needed to determine if patients suffer from pancreatic ductal carcinoma should be tested for VEGF expression before receiving anti-VEGF treatment.

It may also be interesting to note that pancreatic adenocarcinoma cells with advanced tumor stage and poor prognosis, express low level of VEGF receptor, an element required for autocrine action of VEGF. ${ }^{27}$ Additionally, high microvessel density (HMD) in pancreatic adenocarcinomas was found to correlate with increased VEGF expression. ${ }^{27}$ Together, they seem to suggest that for pancreatic ductal adenocarcinomas, the mechanism by which the overexpressed VEGF promotes tumor aggression, is primarily via paracrine pathways and neoangiogenesis.

While this study showed interesting results, limitations exist by its retrospective study design, very small case numbers, and the embedded variability of immunohistochemical methods. Further studies to validate these results are necessary.

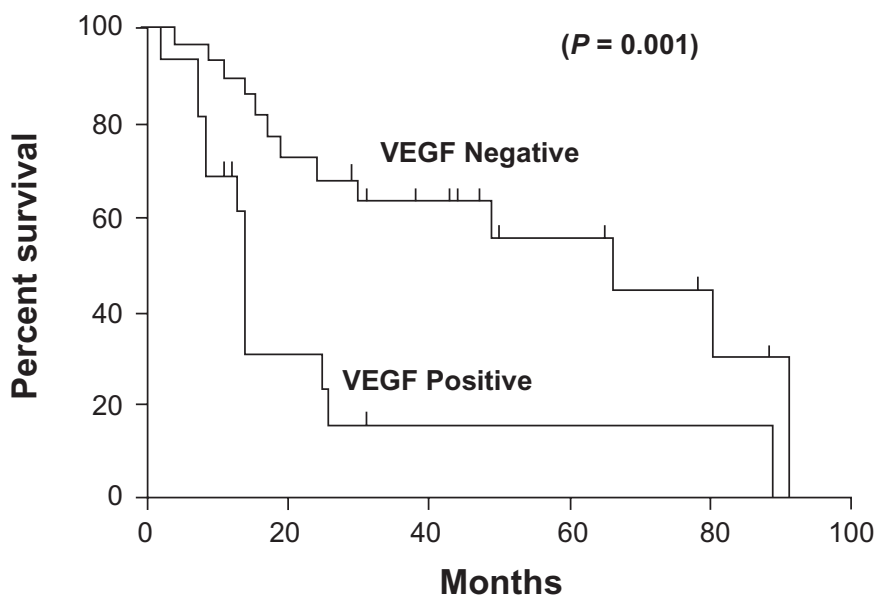

\begin{tabular}{|l|}
\hline Median survival: \\
VEGF negative $=65$ months \\
VEGF positive $=13$ months \\
5 -year survival rate: \\
VEGF negative $=52 \%$ \\
VEGF positive $=17 \%$
\end{tabular}

Figure 4 Kaplan-Meier plots and log-rank tests of survival of patients with VEGF-negative and -positive pancreatic ductal adenocarcinomas. Abbreviation: VEGF, vascular endothelial growth factor. 


\section{Disclosure}

The authors report no conflicts of interest in this work.

\section{References}

1. Jemal A, Siegel R, Ward E, et al. Cancer statistics. CA Cancer JClin. 2007; 57(1):43-66.

2. Warshaw AL, Fernandez-del Castillo C. Pancreatic carcinoma. N Engl J Med. 1992;326(7):455-465.

3. Evans DB, Abruzzese JL, Rich TA. Cancer of pancreas. In: Devita VT, Hellman S, Rosenberg SA (editors). Cancer: Principles and Practice of Oncology. Philadelphia, PA: Lippincott Williams \&Wilkins; 2001.

4. McGrath PC, Sloan DA, Kenady DE. Surgical management of pancreatic carcinoma. Semin Oncol. 1996;23(2):200-212.

5. Yeo TP, Hruban RH, Leach SD, et al. Pancreatic cancer. Curr Probl Cancer. 2002;26(4):176-275.

6. Kleeff J, Michalski C, Friess H, Buchler MW. Pancreatic cancer: from bench to 5-year survival. Pancreas. 2006;33(2):111-118.

7. Farnell MB, Pearson RK, Sarr MG, et al. A prospective randomized trial comparing standard pancreatoduodenectomy with pancreatoduodenectomy with extended lymphadenectomy in resectable pancreatic head adenocarcinoma. Surgery. 2005;138(4):618-628.

8. Marsh RW, George T. Rationale and appropriate use of chemotherapy and radiotherapy for pancreatic ductal adenocarcinoma. Curr Gastroenterol Rep. 2006;8(2):111-120.

9. Saif MW. Anti-angiogenesis therapy in pancreatic carcinoma. JOP. 2006;7(2):163-173

10. Jimeno A, Hidalgo M. Molecular biomarkers: their increasing role in the diagnosis, characterization, and therapy guidance in pancreatic cancer. Mol Cancer Ther. 2006;5(4):787-96.

11. Ferrara N. Vascular endothelial growth factor: basic science and clinical progress. Endocr Rev. 2004;25(4):581-611.

12. Ferrara N. VEGF and quest for tumour angiogenesis factor. Nat Rev Cancer. 2002;2(10):795-803.

13. Hicklin DJ, Ellis LM. Role of the vascular endothelial growth factor pathway in tumor growth and angiogenesis. J Clin Oncol. 2005;23(5): 1011-1027.

14. Breen EC. VEGF in biological control. J Cell Biochem. 2007;102(6): $1358-1367$.
15. Fragoso R, Elias AP, Dias S. Autocrine VEGF loops, signaling pathways, and acute leukemia regularion. Leuk Lymphoma. 2007;48(3):481-488.

16. Lee TH, Seng S, Sekine M, et al. Vascular endothelial growth factor mediates intracrine survival in human breast carcinoma cells through internally expressed VEGFR1/FLT1. PloS Med. 2007;4(6):e186.

17. American Joint Committee on Cancer. Cancer Staging Manual, 6th Edition. Chicago, IL; AJCC; 2002.

18. Jiang Z, Chu PG, Woda BA, et al. Analysis of RNA-binding protein IMP3 to predict metastasis and prognosis of renal cell carcinoma: a retrospective study. Lancet Oncol. 2006;7(7):556-564.

19. Itakura J, Ishiwata T, Friess H, et al. Enhanced expression of VEGF in human pancreatic cancer correlates with local disease progression. Clin Cancer Res. 1997;3(8):1309-1316.

20. Guang-Wu H, Sunagawa M, Jie-En L, et al. The relationship between microvessel density, the expression of VEGF, and the extension of nasopharyngeal carcinoma. Laryngoscope. 2000;110(12):2066-2069.

21. Konno H, Baba M, Kamiya K, et al. Overexpression of VEGF is responsible for hematogenous recurrence of early stage gastric cancer. Eur Surg Res. 2000;32(3):177-181.

22. Seo T, Baba H, Fukuda T, Takashima M, Sugimachi K. High expression of vascular endothelial growth factor is associated with liver metastasis and a poor prognosis for patients with ductal pancreatic adenocarcinoma. Cancer. 2000;88(10):2239-2245.

23. Weidner N, Semple JP, Welch WR, Folkman J. Tumor angiogenesis and metastasis: correlation in invasive breast carcinoma. $N$ Engl J Med. 1991;324(1):1-8

24. Srivastava A, Laidler P, Davies RP, Horgan K, Hughes LE. The prognostic significance of tumor vascularity in intermediate-thickness (0.76-4.0 mm thick) skin melanoma: a quantitative histologic study. Am J Pathol. 1988;133(2):419-423.

25. William WN Jr., Kies MS, Fossella, et al. Phase 2 study of carboplatin, docetaxel, and bevacizumab as frontline treatment for advanced nonsmall-cell lung cancer. Cancer. 2010 Mar 11 [Epub ahead of print].

26. Van Cutsem E, Vervenne WL, Bennouna J, et al. Phase III trial of bevacizumab in combination with gemcitabine and erlotinib in patients with metastatic pancreatic cancer. J Clin Oncol. 2009;27(13):2231-2237.

27. Chung GG, Yoon HH, Zerkowski MP, et al. Vascular endothelial growth factor, FLT-1, and FLK-1 analysis in a pancreatic cancer tissue microarray. Cancer. 2006;106(8):1677-1684.
Pathology and Laboratory Medicine International

\section{Publish your work in this journal}

Pathology and Laboratory Medicine International is a peer-reviewed, open access journal focusing on innovative basic research and translational research related to pathology or human disease. The journal includes original research, updates, case reports, reviews and commentaries on current controversies. The Academic Sponsor

\section{Dovepress}

of this journal is the Chinese American Pathology Association (CAPA). The manuscript management system is completely online and includes a very quick and fair peer-review system. Visit http://www.dovepress.com/testimonials.php to read real quotes from published authors. 